\title{
Radiology and imaging
}

Welcome to the second Radiology and Imaging Journals Watch list. Articles in this list have been selected from material published since January 2005. They represent recommended reading only.

When available the citations are linked to the abstract held on PubMed (Medline) and CrossRef.

1. Strong K, Wald N, Miller A, Alwan A. WHO Consultation Group. Current concepts in screening for noncommunicable disease: World Health Organization Consultation Group Report on Methodology of Noncommunicable Disease Screening [Abstract]. J Med Screen 2005; 12: 12-19.

2. Littrup PJ, Freeman-Gibb L, Aleodor A, White M, Amerikia KC, Bouwman D, Harb T, Sakr W. Cryotherapy for breast fibroadenomas [Abstract]. Radiology 2005; 234: 63-72.

3. Santamaría G, Velasco M, Farré X, Vanrell JA, Cardesa A, Fernández PL. Power Doppler sonography of invasive breast carcinoma: does tumor vascularization contribute to prediction of axillary status? [Abstract]. Radiology 2005; 234: 374-380.

4. Oestreicher N, Lehman CD, Seger DJ, Buist DSM, White E. The incremental contribution of clinical breast examination to invasive cancer detection in a mammography screening programme [Abstract]. Am J Roentgenol 2005; 184: 428-432.

Prepared by

A. Evans

Consultant Radiologist

Breast Screening Unit

City Hospital, Hucknall Road Nottingham NG5 1PB, UK 